DOI: $10.3901 / J M E .2019 .15 .071$

\title{
骨组织工程多孔生物支架设计研究进展
}

\author{
屈华伟 韩振宇 卓 越 富宏亚 \\ (哈尔滨工业大学机电工程学院 哈尔滨 150001)
}

\begin{abstract}
摘要: 由人口老龄化、严重不愈合骨折等引起的骨缺损, 使人们对人工骨替代物的需求急剧增加。支架作为骨缺损的人工骨 替代物, 在骨组织工程新骨再生中起到至关重要的作用。人体不同组织(硬骨和软骨), 甚至是相同组织的不同部位(骨骼的中 部和外部)对支架的力学性能、内部微观结构和孔隙率等参数的要求都不尽相同。如何在理想生物材料的基础上, 模拟天然骨 的性能和结构, 设计出具有可控力学性能、结构和渗透率等性能的支架, 一直困扰着组织工程研究者。综述了多孔骨支架在 设计成型方面近年来国内外代表性的研究工作, 重点探究了多孔支架设计方法, 阐述了多孔支架在骨组织工程应用中面临的 挑战, 为深入开展可控多孔支架的研究和应用提供一定的参考。
\end{abstract}

关键词: 骨缺损; 骨组织工程; 多孔生物支架; 可控性能; 梯度孔隙结构

中图分类号: TH16; Q811

\section{Review of the Design of Porous Bio-scaffolds for Bone Tissue Engineering}

\author{
QU Huawei HAN Zhenyu ZHUO Yue FU Hongya
}

(School of Mechatronics Engineering, Harbin Institute of Technology, Harbin 150001)

\begin{abstract}
Due to the aging of the population, severe nonunion fracture and other bone defects, the demand for artificial bone substitutes has increased dramatically. As an artificial substitute for bone defects, scaffolds play a crucial role in new bone regeneration for bone tissue engineering. Different tissues of the human body (bone and cartilage), and even different parts of the same tissue (the central part and the external part of bone), have different requirements for the mechanical properties, internal microstructure and porosity of the scaffolds. How to simulate the performance and structure of natural bone on the basis of ideal biological materials, design a scaffold with controllable mechanical properties, gradient pore structure and permeability, has been plaguing tissue engineering researchers. The representative research work of porous bone scaffolds in design and molding has been reviewed in recent years. The design methods of porous scaffolds are mainly explored. The challenges of porous scaffolds are expounded in bone tissue engineering applications. The review provides a reference for the further research and application of controllable porous scaffolds.
\end{abstract}

Key words: bone defects; bone tissue engineering; porous bio-scaffolds; controllable properties; gradient pore structure

\section{0 前言}

骨缺损是骨科的一个重要难题之一，每年发生 1500 多万例骨折患者 ${ }^{[1]}$ 。美国每年进行 50 多万例骨 移植手术，全球约 220 万例，这些手术的年费用接 近 25 亿美元 ${ }^{[2]}$ 。组织移植至少从 1660 年开始就被 人类用于组织修复 ${ }^{[3]}$ 。自体骨移植和异体骨移植被 认为是骨修复的有效选择, 但由于供体部位发病率 高、可用骨数量受限和异体移植免疫排斥等问题严 重影响在临床上的应用。为改善组织移植的困境,

20190119 收到初稿, 20190420 收到修改稿
身兼美国哈佛大学和麻省理工学院教授的 LANGER 等 ${ }^{[4]}$ 于 20 世纪 90 年代初在《Science》上提出组织 工程的概念, 即在可生物降解的人工多孔支架内植 入生长因子、细胞和(或)蛋白质等，用于刺激组织 修复再生。理想的骨支架应具有生物活性、生物降 解性、生物相容性、机械支撑、良好的孔隙率等 特性和物质传递的能力, 而且随着新生骨组织的 增殖生长支架逐渐降解, 直至完全替代支架长成 新骨 ${ }^{[5-9]}$ 。2015 年, 我国第四军医大学的裴国献教 授等 ${ }^{[10]}$ 成功完成世界首例组织工程大段骨缺损修 复再生手术。随着新型生物材料组成的深入研究和 3D 打印技术的日臻成熟, 生物支架发展潜力巨大。 GIANNITELLI 等 ${ }^{[11]}$ 综述了组织工程支架的部分设 
计方法和支架设计所面临的挑战。 $\mathrm{YOO}^{[12]}$ 就 $\mathrm{CAD}$ 在组织工程的发展趋势和面临的挑战进行了总结。 本文从支架成型的角度出发, 综述了多孔支架设计 方法在骨组织工程的最新进展。

\section{1 骨组织工程生物支架的研究进展}

材料组成是影响支架各项性能的关键参数之 一。天然高分子材料(胶原蛋白、明胶、壳聚糖等) 具有良好的生物相容性和丰富的细胞识别位点, 但 力学性能普遍偏低; 合成高分子材料力学性能优异, 但生物活性不足。研究发现由两种或两种以上材料 组成的支架材料具有更好的力学性能和生物活 性 ${ }^{[13-19]}$ 。近年来国内外研究者在支架材料方面取得 众多进展 ${ }^{[20-25]}$ 。美国华盛顿大学的 BOSE 等 ${ }^{[26]}$ 研究 了覆盖 PCL 的 TCP 支架在体内成骨的影响。印度 理工学院的 MISHRA 教授等 ${ }^{[27]}$ 制备了壳聚糖/水凝 胶/海藻酸盐/n-HAp 的纳米生物复合支架。西安交 通大学的李涤尘教授等 ${ }^{[28-30]}$ 制备了用于肝脏组织工 程的壳聚糖/明胶复合支架和用于软骨组织工程的 $\beta-\mathrm{TCP} /$ 明胶支架。

在研究各种生物材料使支架具有理想的生物相 容性和生物可降解性的基础上, 支架还须具备良好 的力学性能、孔隙结构和渗透率等 ${ }^{[31-32]}$ 。适宜的力 学性能是支架建立三维生长环境供新生骨组织增
殖、生长的关键。支架力学性能过低将不能支撑起 组织细胞生存的三维环境, 过高将产生应力屏蔽效 应 $^{[33-38]}$ 。由于人体缺损部位的不同，相同材料的支 架所需的力学性能、梯度孔隙结构也不相同。合理 的孔隙结构可以调整支架所需的不同力学性能。

BELLER 等 ${ }^{[39]}$ 构建了人体椎骨 ESA29-99-L3 的三维 微观结构, 如图 1a 所示, 发现天然骨具有明显的梯 度孔隙结构, 骨骼具有中部孔隙率高于外部的特点。

为模拟天然骨的内部结构, 理想支架应具有互 相连通的梯度孔隙结构, 允许蛋白吸附、细胞扩散 和营养物质、氧气、生长因子和废物等的有效运输, 促进新生骨组织在支架内部的生长, 直至支架完全 降解并建立新骨以达到修复骨缺损的终极目标。在 国外, 西班牙加泰罗尼亚理工学院的 Fernández 教 授等 ${ }^{[40]}$ 通过 Voronoi 镶嵌法得到具有各向同性的骨 小梁三维多孔模型, 其主要组织形态学指标与天然 骨小梁完全吻合，如图 1b 所示。印度理工学院的 BALANI 等 ${ }^{[41]}$ 研究发现通过控制支架孔隙分布,

HAp 生物陶瓷复合支架的力学性能和生物性能均 得到显著提高。在国内, 西安交通大学的李涤尘教 授等 ${ }^{[42]}$ 提出基于 $3 \mathrm{D}$ 打印的可变模量的金属假体微 结构设计，设计的多孔微结构有效改善了应力屏蔽 效应。南京航空航天大学的赵剑锋教授等 ${ }^{\left[{ }^{[3]}\right.}$ 基于 Voronoi 镶嵌法研究了可控不规则多孔支架的设计, 获得了具有特定功能梯度孔隙的多孔结构。

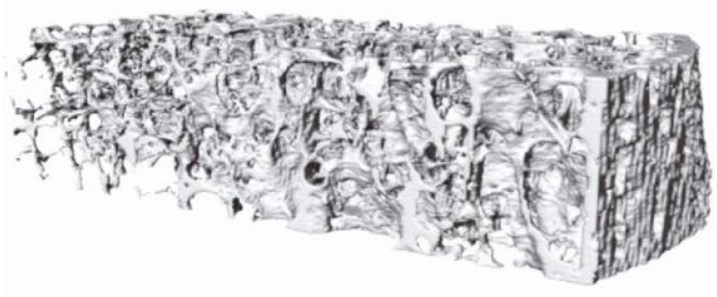

(a) 人体椎骨 $3 \mathrm{D}$ 结构

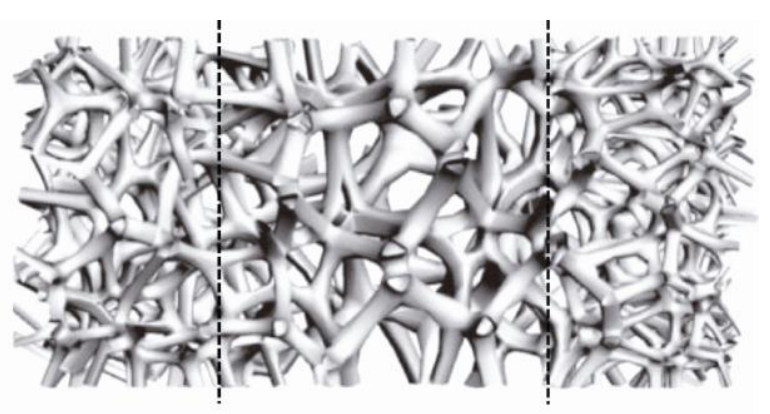

(b) 具有孔隙梯度的多孔支架

图 1 梯度孔隙结构

近年来，随着增材制造技术和 CAD/CAM 技术 的日鉌成熟, 实现复杂可控多孔支架的设计与制造 成为可能。增材制造(Additive manufacturing, AM), 也称为快速成型 (Rapid prototyping, RP)或固体自由 制造(Solid free-form fabrication, SFF)技术出现于 20 世纪 80 年代末, 解决了传统技术无法制造复杂结构 的难题 ${ }^{[44-49]}$ 。增材制造技术主要包括 3D 打印 (Three-dimensional printing, 3DP)、立体光刻 (Stereolithography, SLA)、选择性激光烧结(Selective laser sintering, SLS)、熔融沉积成型(Fused deposition modelling, FDM)和低温沉积制造(Low-temperature deposition manufacturing，LDM)等。增材制造技术 可根据患者特异性要求定制仿生支架, 极大地促进 了复杂多孔支架在骨组织工程的发展。其中，清华 大学王小红教授等 ${ }^{[50-52]}$ 研发了国内第一台细胞 3D 打印机，以明胶/纤维蛋白原为通用基质材料适用于 大部分的细胞组装。清华大学的熊卓博士通过精密 喷射制造技术(Precise extrusion manufacturing, PEM) 制备 PLLA 多孔支架 ${ }^{[53]}$ 。

由于骨组织在整个生存周期是动态变化的, 导 致其多孔结构、力学性能等参数也在跟随变化。在 实现复杂生物支架制造成型的基础上，多孔支架设 
计的关键是在给定尺寸上, 所构建的骨支架替代物 不仅应具有与天然骨相似的内部结构, 而且应具有 可控的孔隙率、梯度孔隙结构、力学性能等参数。 本文主要介绍标准多面体的布尔运算、拓扑优化、 隐式曲面(Implicit surface)、Voronoi 镶嵌法(Voronoi tessellation method)、医学图像设计法和成型后体外 处理法在多孔生物支架结构设计中的应用, 并针对 不同设计方法进行了总结归纳, 以期为组织工程研 究者提供灵感和思路, 为人工支架最终在临床上广 泛应用提供一定借鉴意义。

\section{2 多孔生物支架的设计方法}

\section{1 标准多面体的布尔运算}

早期多孔支架的研究是将标准多面体(如立方 体、球、圆柱和六边形等)通过布尔运算构建单元晶 胞, 再对构建的单元晶胞进行阵列创建三维模型。 为了减少设计的操作时间, 新加坡南洋理工大学的 CHUA 教授等 ${ }^{[54-55}$ 提出基于标准多面体的组织支架 计算机辅助系统(Computer-aided system for tissue scaffolds, CASTS)的模型库, 部分单元晶胞和单元 晶胞阵列后的支架如图 2 所示。

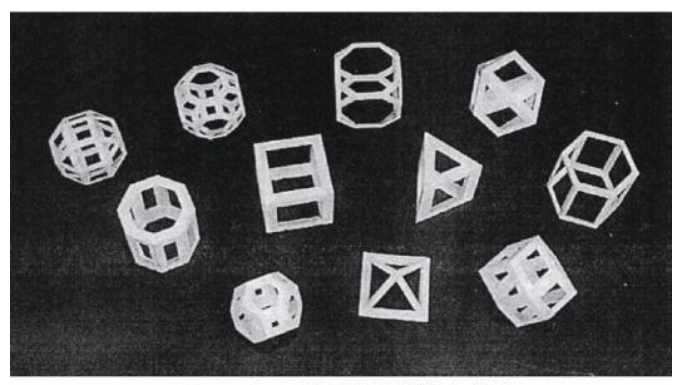

(a) CASTS 模型库的单元晶胞

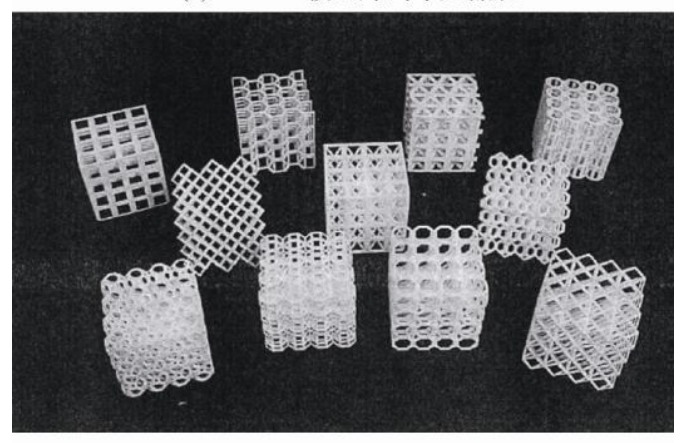

(b) CASTS 模型库单元晶胞的阵列

图 2 CASTS 模型库

经 CASTS 模型库设计, 借助 SLS 技术所制备 的聚酰胺(Polyamide, PA)支架孔隙率较大, 其力学 性能和渗透性较差, 难以满足天然骨组织生长环境 的要求 ${ }^{[55]}$ 。该方法作为早期多孔支架设计的尝试, 只是进行简单的布尔运算和阵列, 建立了 CASTS
模型库，且所制支架的孔隙率、梯度孔隙等参数可 控性很差。但该方法在一定程度上为组织工程发展 提供了一些灵感和思路。未来可以优秀的支架设计 方法和性能优异的生物材料为基础, 建立骨组织工 程数据库, 进行资源共享, 可有效提高研究人员学 习工作的效率。

\section{2 拓扑优化}

拓扑优化基本均是基于均匀化理论, 可通过相 关物理量的渐进展开式产生多尺度均衡方程, 用于 计算基于单元晶胞的有效性质。弹性模量的均质化 分析可以跨层次尺度递归应用, 以计算从最小特性 到最大特性(整体支架弹性模量)的影响。美国密歇 根大学的 HOLLISTER 教授 ${ }^{[56]}$ 提出基于计算机拓扑 设计(Computational topology design, CTD)和 SFF 技 术定制多孔支架的想法, 并制备了 PCL 材料的下领 骨髁突骨支架, 测试可得支架机械性能满足设计的 要求。如图 3 所示为所设计的球孔和柱孔单元晶胞。

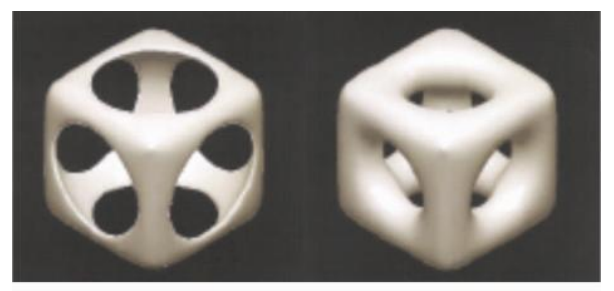

(a) 球孔

(b) 柱孔

图 3 拓扑优化模型

通过求解单元晶胞在六种局部应变状态下的变 形, 进而由微观层次 $C_{i j p m}^{\text {micro }}$ 计算出宏观层次的有效模 量 $C_{i j k l}^{\text {macro }}$, 可得

$$
C_{i j k l}^{\text {macro }}=\frac{1}{\left|V_{\text {unit-cell }}\right|} \int_{V} C_{i j p m}^{\text {micro }} M_{p m k l} \mathrm{~d} V_{\text {unit-cell }}
$$

式中, $M_{p m k l}$ 为局部张量; $V_{\text {unit-cell }}$ 为单元晶胞体积。

对于物料运输, 宏观渗透率 $K_{i j}^{\text {macro }}$ 可以通过斯 托克斯流速矢量 $v_{j}^{i}$ 来计算, 如下式

$$
K_{i j}^{\text {macro }}=\frac{1}{\left|V_{\text {unit-cell }}\right|} \int_{V} v_{j}^{i} \mathrm{~d} V_{\text {unit-cell }}
$$

其中, $v_{j}^{i}$ 矢量可由三个单独应用的单位压力梯度求得。

试验结果验证了增加支架材料的用量可以增加 弹性性能, 但降低了支架的渗透率。对于相同孔隙 率下的支架，不同的微观结构将产生不同的有效刚 度和渗透率。通过该方法设计的具有微观结构的支 架, 可以实现与天然骨具有相同的弹性模量, 且支 架具有更高的渗透率以促进细胞迁移和物料运输。 但拓扑优化设计方法计算变量多、运算量大，如何 平衡结构设计和计算效率的关系尚待解决。此外, 拓扑优化设计没有考虑支架的尺寸效应。 


\section{3 隐式曲面}

隐式曲面使用三角函数来优化设计梯度孔隙结 构和最大化支架的比表面积, 这对细胞迁移和组织 内向生长有积极影响 ${ }^{[57-59]}$ 。三周期极小曲面(Triply periodic minimal surface, TPMS)作为隐式曲面的一 种, 具有理想的连通性和可控性等优点。FENG 等 ${ }^{[60]}$ 通过 SLA 技术制备了基于 $\mathrm{T}$ 样条实体的 TPMS 多孔支架, 证明了该种方法的有效性和灵活性。

图 4 展示了在 $85 \%$ 孔隙率下, 隐式曲面优化生
成的部分支架和它们旋转 $45^{\circ}$ 后的支架模型 ${ }^{[40]}$ 。其 中，支架的单元晶胞分别由 Neovius 极小曲面 (Neovius surface, NS), Schwarz 原始曲面(Schwarz Primitive, SP), Schwarz 钻石曲面(Schwarz Diamond, SD), Schoen 螺旋二十四面体曲面(Schoen Gyroid,

SG)和 Schwarz 的 W 曲面(Schwarz 'W', SW)优化构 成。对上述支架进行测试分析显示, 旋转 $45^{\circ}$ 后制 备的支架与未旋转的支架相比，具有各向异性的力 学性能和流体流动性能。

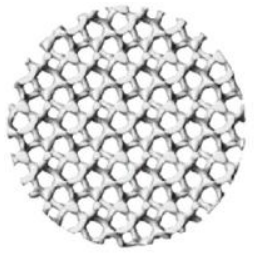

NS

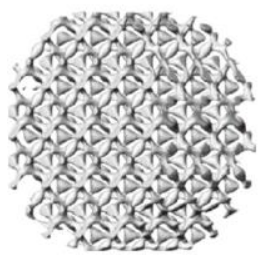

$\mathrm{NS}-45^{\circ}$

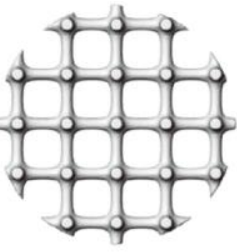

SP

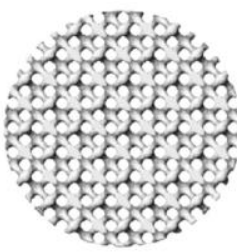

SD

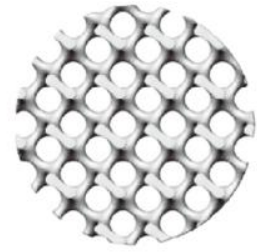

SG

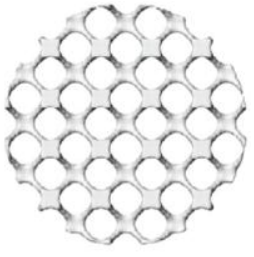

SW

(a) 隐式曲面的几种三维多孔模型

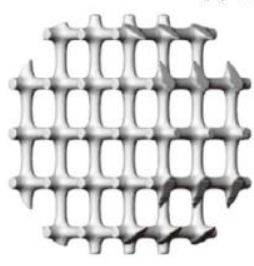

$\mathrm{SP}-45^{\circ}$

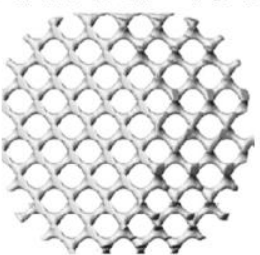

$\mathrm{SD}-45^{\circ}$

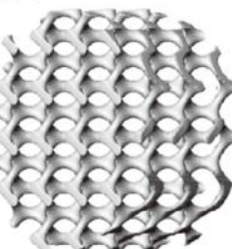

$\mathrm{SG}-45^{\circ}$

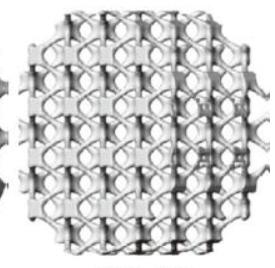

$\mathrm{SW}-45^{\circ}$

(b) 旋转 $45^{\circ}$ 后隐式曲面的几种三维多孔模型

图 4 隐式曲面多孔模型

隐式曲面虽能提供各向异性的性能, 但其特定 的隐式函数只能描述一定范围的支架结构, 存在没 有隐式函数与目标支架相对应的情况, 限制了隐式 函数对全部多孔支架的准确描述。

\subsection{Voronoi 镶嵌法}

Voronoi 镶嵌法是将患者医学数据生成 $2 \mathrm{D}$ Voronoi 单元结构, 再将其生成 3D Voronoi 模型, 以完成模型的重建。以人体骨小梁为例, 将骨小梁 的微观计算机断层扫描 (Micro computed tomography, $\mu \mathrm{CT}$ ) 数据通过软件 Image $^{\circledR}{ }^{\circledR} \mathrm{v} .1 .47$ 转换 为 $2 \mathrm{D}$ Voronoi 点坐标 $(x, y)$, 以平均骨小梁间距为 $\mathrm{z}$ 等距处理, 构建 3D Voronoi 点坐标 $(x, y, z)$, 通过 3D Voronoi Grasshopper 软件构建 3D Voronoi 单元 晶胞多孔结构 ${ }^{[40]}$, 具体过程如图 5 所示。

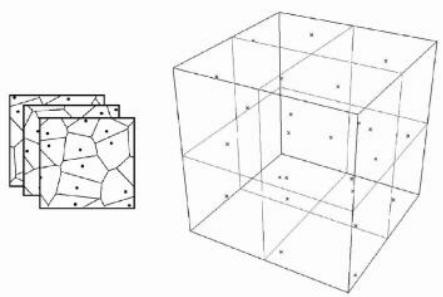

(a)

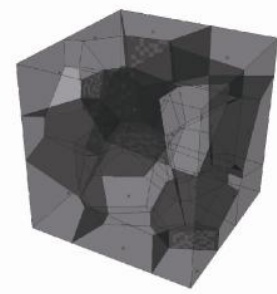

(b)

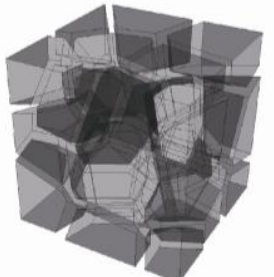

(c)

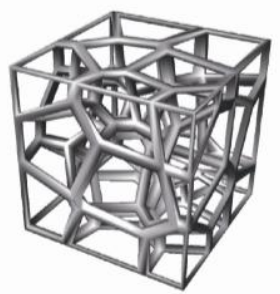

(e)

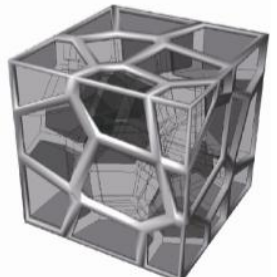

(d)

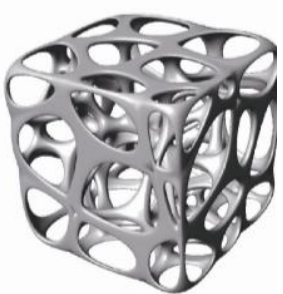

(f)
图 53 D Voronoi 单元晶胞多孔结构的设计过程

在 Voronoi 单元晶胞成核点数 (Number of nucleating points, NNP) 一定的情况下, 可以通过调 整骨小梁间距和骨小梁厚度，实现控制 Voronoi 单 元晶胞孔隙率的目的 ${ }^{[40]}$ (图 6a); 在孔隙率一定的情 况下，可通过调整成核点数，实现控制 Voronoi 单 元晶胞的比表面积 ${ }^{[40]}$ (图 6b)。通过控制成核点数、 孔隙率、骨小梁间距和骨小梁厚度等参数, 并结合 
梯度孔隙结构可设计出具有广泛参数的支架模型。

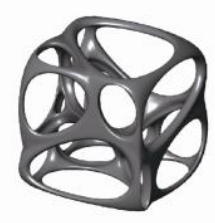

$\mathrm{NNP}=5 ; \Phi(\%)=89$

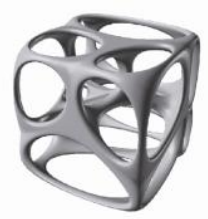

$\mathrm{NNP}=5 ; \Phi(\%)=89$

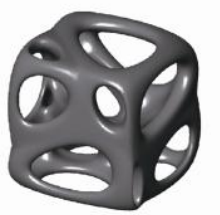

$\mathrm{NNP}=5 ; \Phi(\%)=69$

(a)

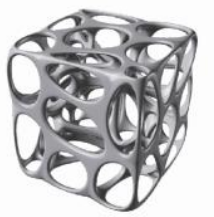

$\mathrm{NNP}=25 ; \Phi(\%)=89$

(b)
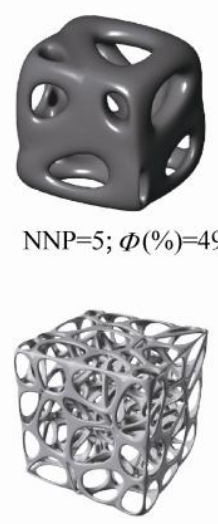

$\mathrm{NNP}=50 ; \Phi(\%)=89$
$\mathrm{NNP}=5 ; \Phi(\%)=49$

图 6 不同成核点数 $(\mathrm{NNP})$ 和孔隙率 $(\Phi)$ 下的单元晶胞

如图 7 所示为成核点数为 $10 、 100$ 和 125 的 Voronoi 单元晶胞与几种常见的隐式曲面单元晶胞 孔隙率与比表面积(Bone surface to bone volume, $\mathrm{BS} / \mathrm{BV})$ 的关系 ${ }^{[00]}$ 。可以发现在比表面积一定的情况 下, 不同成核点数的 Voronoi 模型具有不同的孔隙率, 但隐式曲面模型特定函数只可对应唯一的孔隙率。

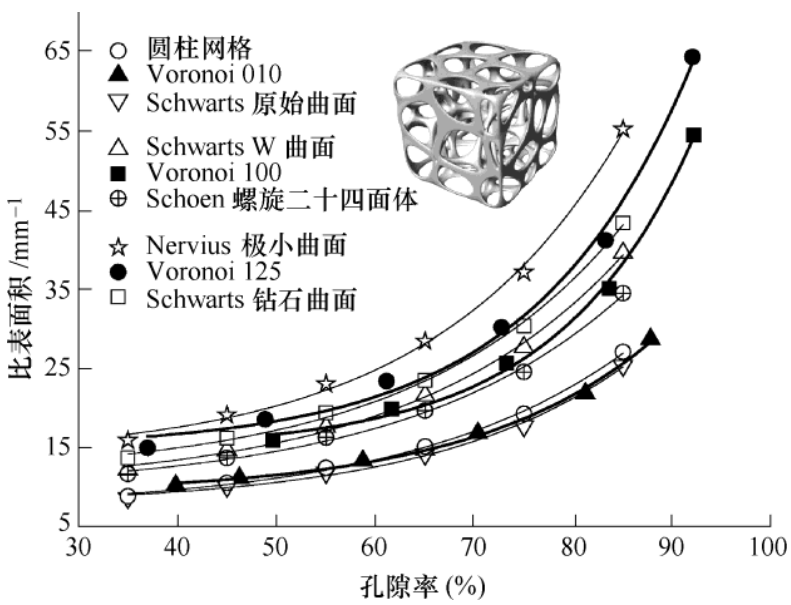

图 7 不同单元晶胞孔隙率和比表面积的关系
Voronoi 模型基本覆盖了常见的隐式曲面模 型, 可表达范围更广的支架模型。通过 Voronoi 镶 嵌法可设计出具有不同孔隙率、梯度孔隙结构、 比表面积等参数的 Voronoi 单元晶胞, 可以实现对 支架梯度孔隙、孔隙分布和孔隙形状等参数的精 确控制。赵剑锋教授 ${ }^{[3]}$ 通过 Voronoi 镶嵌法设计了 与天然骨具有相同内部结构的 CAD 模型(图 8), 并通过选择性激光熔融技术制备了金属支架。但 对于非金属生物材料来制备这类支架依然存在一 定技术困难。

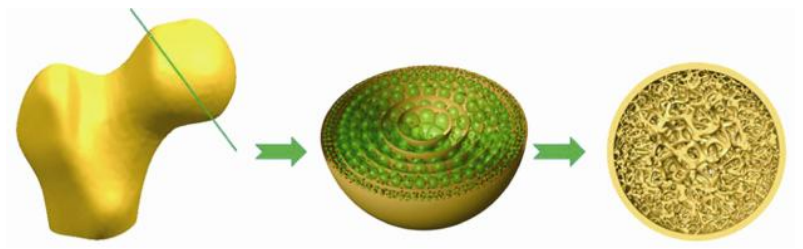

图 8 人工骨的多孔梯度结构重建

\section{5 医学图像设计法}

根据计算机断层扫描(Computed tomography, CT)、 $\mu \mathrm{CT}$ 和核磁共振成像 (Magnetic resonance imaging, MRI)等医学成像技术获得患者骨缺损的原 始数据, 通过包括 3D 图像处理在内的计算机辅助 设计(Computer aided design, $\mathrm{CAD}$ )技术重建患处的 $3 \mathrm{D}$ 模型。YAO 等 ${ }^{[16]}$ 基于 $\mathrm{CT}$ 数据重建了新西兰白 兔股骨和椎板骨支架模型, 借助 FDM 技术制造了 PCL/HAp 多孔支架。BELLER 等 ${ }^{[39]}$ 通过 980 张分辨 率为 2048 像素 $\times 2048$ 像素的人体骨椎 $\mu \mathrm{CT}$ 图片重 建了人体骨椎模型, 如图 9 所示。由于直接采用原 始医学数据资料, 医学图像设计法可相对准确地重 建天然骨的三维模型, 特别是随着采集数据的精确 化, 重建的模型将更加准确。但图像带来的噪声、 数据集的维度和物理分辨率在一定程度上限制了医 学图像设计法的应用。

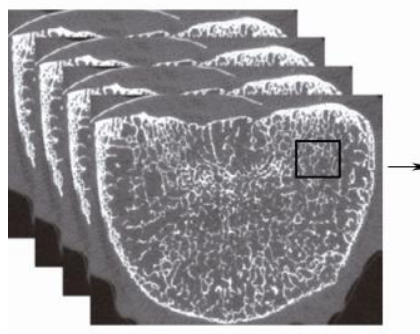

患者椎体连续 $\mu \mathrm{CT}$ 图片

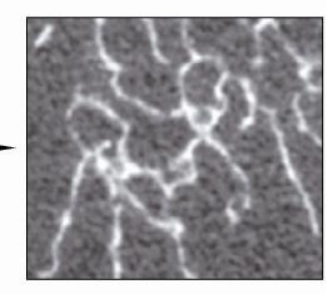

二值化图像处理

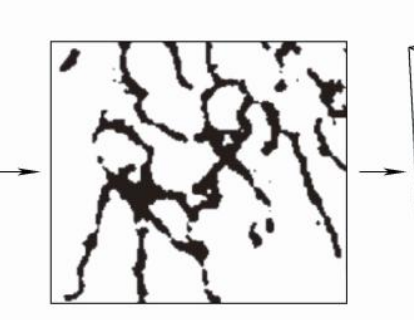

平滑图像处理

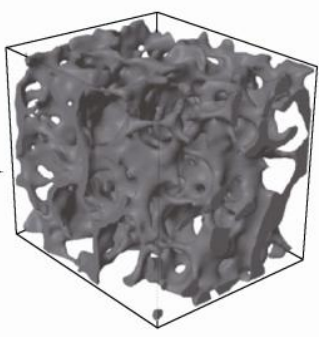

3D 模型重建

图 9 医学图像设计法重建椎体模型

\section{6 成型后体外处理法}

韩国朝鲜大学的 KIM 教授等 ${ }^{[19]}$ 通过 $3 D$ 打印制 备的 PLGA/n-HAp/ $/$-TCP 支架, 经刻蚀氧等离子表 面改性处理后增加了支架表面亲水性和粗䊁度, 如
图 10 所示。经该方法得到的支架进行体外骨细胞 (MC3T3-E1)培养试验, 结果表明该处理方法对细胞 粘附、增殖和分化有积极影响, 验证了刻蚀技术对 提高骨组织工程生物相容性的积极作用。 


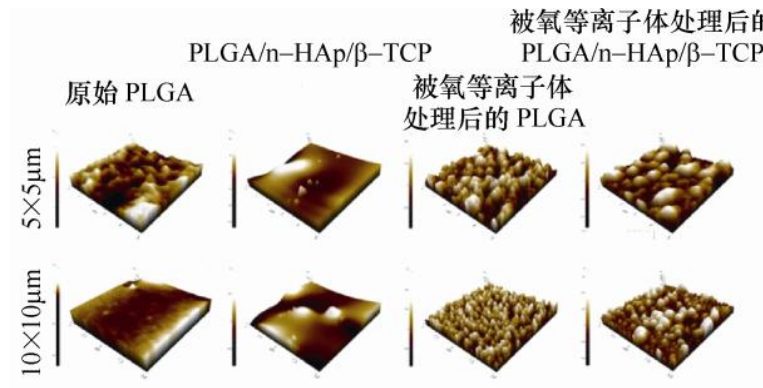

(a)

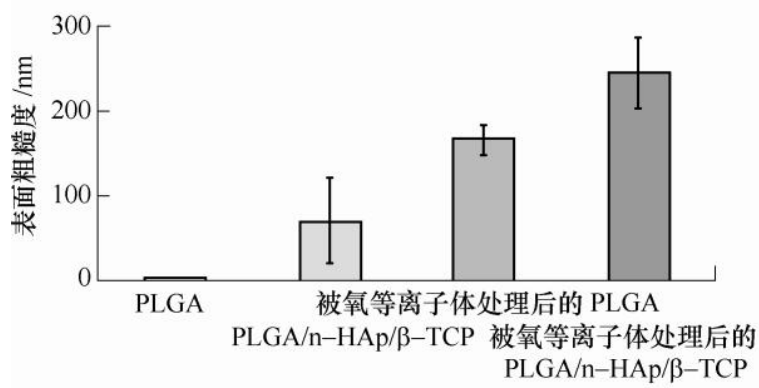

(b)

图 10 刻蚀前后支架表面 $\mathrm{AFM} 3 \mathrm{D}$ 图像和粗粘度 $R q$

深圳大学的罗永祥等 ${ }^{[61]}$ 基于 3D 打印和原位矿 化技术制备具有纳米磷灰石涂层的海藻酸盐/明胶 复合支架, 与对照组相比, 具有纳米磷灰石涂层支 架的力学性能和蛋白吸附均得到显著性提高, 如下 图 11 所示。通过研究总结上述文献和类似文献[6263]中支架成型之后的处理方法, 提出并定义了 “成
型后体外处理法” 的概念。即支架成型后, 通过合 适的方式(如物理刻蚀或化学反应等)可实现在体外 对支架特征的二次处理, 增加或提高支架某些性能。 该发现将这类处理方式归为一个概念，丰富了组织 工程的内容，有望提高研究人员的工作效率。

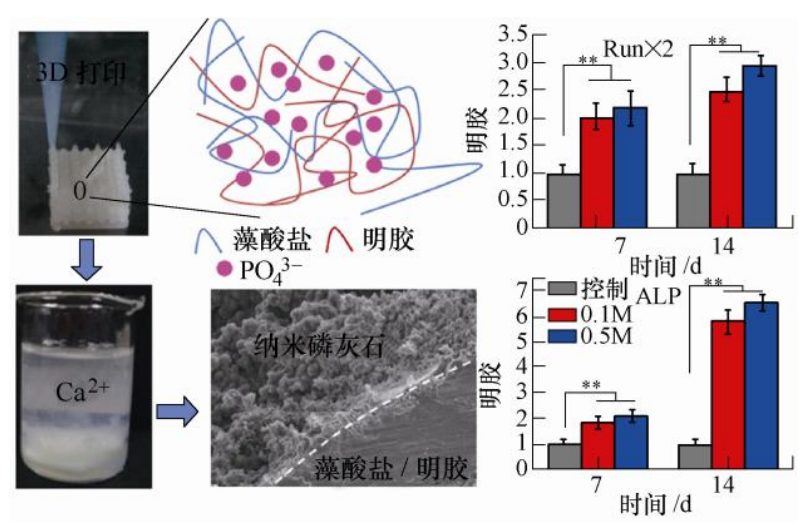

图 11 基于原位矿化技术的支架纳米磷灰石涂层

\section{3 多孔支架设计方法的比较和讨论}

各种多孔支架设计方法，均有其优势和不足， 表 1 从设计原理、结构、力学性能、渗透率、材料、 成本、效率和优缺点方面对上述 6 种设计方法进行 了比较。

表 1 多孔支架设计方法的比较

\begin{tabular}{|c|c|c|c|c|c|c|}
\hline 方法/内容 & $\begin{array}{l}\text { 标准多面体的 } \\
\text { 布尔运算 }\end{array}$ & 拓扑优化 & 隐式曲面 & Voronoi 镶嵌法 & 医学图像设计法 & $\begin{array}{c}\text { 成型后体外 } \\
\text { 处理法 }\end{array}$ \\
\hline 设计原理 & $\begin{array}{l}\text { 标准多面体的布尔运 } \\
\text { 算, 所生成的单元晶胞 } \\
\text { 再进行阵列 }\end{array}$ & $\begin{array}{l}\text { 基于均质化理论计 } \\
\text { 算支架弹性模量和 } \\
\text { 渗透率 }\end{array}$ & $\begin{array}{l}\text { 三角函数来最大化 } \\
\text { 支架的比表面积 }\end{array}$ & $\begin{array}{l}\text { 由医学数据生成 } 2 \mathrm{D} \\
\text { Voronoi 单元, 再构 } \\
\text { 建 } 3 \mathrm{D} \text { Voronoi }\end{array}$ & $\begin{array}{l}\text { 通过 CAD 技术处理 } \\
\text { 患者医学数据 }\end{array}$ & $\begin{array}{l}\text { 物理处理或化学反 } \\
\text { 应处理 }\end{array}$ \\
\hline 结构 & $\begin{array}{l}\text { 标准多面体的 } \\
\text { 布尔集合 }\end{array}$ & $\begin{array}{l}\text { 几何形状的 } \\
\text { 表面优化 }\end{array}$ & 特殊曲面的优化 & $\begin{array}{l}\text { 可控孔隙率和梯度 } \\
\text { 孔隙 }\end{array}$ & 与天然骨基本一致 & $\begin{array}{l}\text { 可增加支架表面的 } \\
\text { 微孔结构等 }\end{array}$ \\
\hline 力学性能 & 较差 & 好 & 好 & 较好 & 较好 & 降低支架学性能 \\
\hline 渗透率 & 较差 & 好 & 好 & 较好 & 较好 & 增加支架的渗透率 \\
\hline 材料 & $\mathrm{PA}$ & PCL & 光敏树脂 & 金属 & PCL/HAp & 有限制 \\
\hline 成本 & 低 & 低 & 低 & 高 & 特别高 & 低 \\
\hline 效率 & 高 & 低 & 高 & 高 & 低 & 高 \\
\hline 优点 & 效率高, 成本低 & $\begin{array}{l}\text { 孔隙率可控、渗透率 } \\
\text { 高、与天然骨相同的 } \\
\text { 弹性模量 }\end{array}$ & $\begin{array}{l}\text { 最大化支架的比表 } \\
\text { 面积、拥有各向异性 }\end{array}$ & $\begin{array}{l}\text { 可模拟天然骨梯度 } \\
\text { 孔隙结构、参数可 } \\
\text { 控性好 }\end{array}$ & $\begin{array}{l}\text { 与天然骨最接近的 } \\
\text { 内部结构 }\end{array}$ & 提高支架性能 \\
\hline 缺点 & $\begin{array}{l}\text { 参数可控性差、孔隙率 } \\
\text { 大、力学性能和渗透性 } \\
\text { 较差 }\end{array}$ & $\begin{array}{l}\text { 涉及变量多、计算量 } \\
\text { 大、没考虑尺寸效应 }\end{array}$ & 函数覆盖小 & $\begin{array}{l}\text { 针对非金属生物材 } \\
\text { 料制造存在一定困 } \\
\text { 难 }\end{array}$ & 成本较高 & $\begin{array}{l}\text { 只可用于支架成型 } \\
\text { 后, 且对材料和反应 } \\
\text { 有要求 }\end{array}$ \\
\hline 参考文献 & [54-55] & [56] & {$[40,57-60]$} & {$[40,43]$} & {$[16,39]$} & [19,61-63] \\
\hline
\end{tabular}

结果显示, 标准多面体的布尔运算设计方法属 于早期多孔支架的探索, 虽构建了 CASTS 模型库, 具有较高的设计效率, 但所制备的支架力学性能和 渗透率都较差, 且关键参数可控性差。拓扑优化设 计的支架具有优秀的孔隙结构和力学性能, 但涉及
变量多, 运算量较大, 如何平衡设计和效率的关系 仍存在挑战, 且没有考虑尺寸效应。隐式曲面具有 各向异性的力学和流体特性, 但其函数描述支架范 围较窄, 函数覆盖小。医学图像设计法因其直接对 天然骨医学信息进行图像处理重建模型结构, 具有 
与天然骨最接近的内部结构, 但是医学数据集的维 度、物理分辨率和图像噪声在一定程度上限制了其 应用。成型后体外处理法可通过对成型后的支架在 体外进行二次处理, 有效提高支架某些特定的性能, 但只能对成型后的支架进行处理, 且对材料和反应 等有限制, 应用领域相对较窄。Voronoi 镶嵌法具有 模拟天然骨孔隙结构的优点, 且可精确控制支架的 梯度孔隙、孔隙形状和孔隙分布等参数。现有技术 虽可制造其金属材料支架, 但对非金属生物材料支 架的制备技术亟待突破, 随着 3D 打印制造技术的 进一步突破, Voronoi 镶嵌法未来发展潜力巨大。

尽管多孔支架在结构设计方面已取得重大进展 和突破，但仍面临以下挑战。

（1）由于生物材料的广泛性, 且不同组织对力 学性能、梯度孔隙结构等参数的要求也不尽相同, 因此如何平衡支架材料、力学性能和孔隙结构设计 的关系显的尤为重要。

(2) Voronoi 镶嵌法在多孔设计领域受到广泛关 注, 其虽可精确设计出具有梯度孔隙等参数的支架, 但与其设计技术想匹配的宏、微观制造成型技术尚 待突破。

(3) 对于支架结构的设计是否越接近天然骨孔 隙结构越理想? 较高孔隙结构或者较大比表面积的 支架是否更有利于组织的再生? 仍然有很多组织工 程基础难题等待研究者去解决。

\section{4 结论与展望}

文章综述了多孔支架的设计方法, 主要得到以 下结论与展望。

(1) 总结并提出 “成型后体外处理法” 的概念。 丰富了组织工程的内容, 有望提高研究人员的工作 效率。

（2）上述众多方法均可设计多孔支架，但真正 能够模拟天然骨梯度孔隙结构的只有 Voronoi 镶嵌 法和医学图像设计法。在大力发展这两种多孔支架 设计方法的同时, 仍须深入探究更优秀的设计方法。

(3) 未来可将两种或多种设计方法相结合, 有 望制备性能更优的支架。如, 若将 Voronoi 镶嵌法 和成型后体外处理法相结合, 支架不仅可以具有与 天然骨相似的内部结构, 而且可使支架拥有优异的 特性以促进蛋白吸附和细胞增殖等。

(4) 未来可借助如多喷头打印等先进制造技术 构建支架材料、内部结构和制造一体化设计, 综合 考虑影响支架性能的各种因素, 为人工支架广泛应 用于临床奠定基础。

\section{参 考 文 献}

[1] GOMEZ S, VLAD M D, LOPEZ J, et al. Design and properties of 3D scaffolds for bone tissue engineering[J]. Acta Biomaterialia, 2016, 42(2016): 341-350.

[2] GIANNOUDIS P V, DINOPOULOS H, TSIRIDIS E. Bone substitutes : An update[J]. Injury-International Journal of the Care of the Injured, 2005, 36(2005): 20-27.

[3] SANAN A, HAINES S J. Repairing holes in the head: A history of cranioplasty[J]. Neurosurgery, 1997, 40(3): 588-603.

[4] LANGER R, VACANTI J P. Tissue engineering[J]. Science, 1993, 260(5110): 920-926.

[5] JONES J R, EHRENFRIED L M, HENCH L L. Optimising bioactive glass scaffolds for bone tissue engineering[J]. Biomaterials, 2006, 27(7): 964-973.

[6] LEE S H, SHIN H. Matrices and scaffolds for delivery of bioactive molecules in bone and cartilage tissue engineering[J]. Advanced Drug Delivery Reviews, 2007, 59(4-5): 339-359.

[7] LEONG K F, CHEAH C M, CHUA C K. Solid freeform fabrication of three-dimensional scaffolds for engineering replacement tissues and organs[J]. Biomaterials, 2003, 24(13): 2363-2378.

[8] LI W J, LAURENCIN C T, CATERSON E J, et al. Electrospun nanofibrous structure: A novel scaffold for tissue engineering $[\mathrm{J}]$. Journal of Biomedical Materials Research, 2002, 60(4): 613-621.

[9] YANG S F, LEONG K F, DU Z H, et al. The design of scaffolds for use in tissue engineering. Part 1. Traditional factors[J]. Tissue Engineering, 2001， 7(6): 679-689.

[10] 刘勇. 成人胫骨平台骨折临床特征和骨折分型系统评 价一致性研究[D]. 西安：第四军医大学, 2017.

LIU Yong. Research on the clinical characteristics of adult tibial plateau fractures and evaluation consistency of fracture classification systems[D]. Xi'an: Fourth Military Medical University, 2017.

[11] GIANNITELli S M, ACCOTO D, TROMBETTA M, et al. Current trends in the design of scaffolds for computer-aided tissue engineering $[\mathrm{J}]$. Acta Biomaterialia, 2014, 10(2): 580-594.

[12] YOO D J. Recent trends and challenges in computer-aided design of additive manufacturing-based biomimetic scaffolds and bioartificial organs[J]. International Journal of Precision Engineering and Manufacturing, 2014, 15(10): 2205-2217.

[13] PARK S A, LEE S J, SEOK J M, et al. Fabrication of 3D printed PCL/PEG polyblend scaffold using rapid prototyping system for bone tissue engineering 
application[J]. Journal of Bionic Engineering, 2018, 15(3): 435-442.

[14] LUO W F, ZHANG S Y, LAN Y W, et al. 3D printed porous polycaprolactone/oyster shell powder (PCL/OSP) scaffolds for bone tissue engineering[J]. Materials Research Express, 2018, 5(4): 1-9.

[15] WANG Y N, WANG K, LI X P, et al. 3D fabrication and characterization of phosphoric acid scaffold with a HA/beta-TCP weight ratio of 60: 40 for bone tissue engineering applications[J]. PLoS One, 2017, 12(4): 1-17.

[16] YAO Q Q, WEI B, GUO Y, et al. Design, construction and mechanical testing of digital 3D anatomical data-based PCL-HA bone tissue engineering scaffold[J]. Journal of Materials Science-Materials in Medicine, 2015, 26(1): 1-9.

[17] SADIASA A, NGUYEN T H, LEE B T. In vitro and in vivo evaluation of porous PCL-PLLA 3D polymer scaffolds fabricated via salt leaching method for bone tissue engineering applications[J]. Journal of Biomaterials Science-Polymer Edition， 2014， 25(2): 150-167.

[18] PARK S A, LEE S H, KIM W D. Fabrication of porous polycaprolactone/hydroxyapatite (PCL/HA) blend scaffolds using a 3D plotting system for bone tissue engineering $[\mathrm{J}]$. Bioprocess and Biosystems Engineering, 2011, 34(4): 505-513.

[19] ROH H S, JUNG S C, KOOK M S, et al. In vitro study of 3D PLGA/n-HAp/beta-TCP composite scaffolds with etched oxygen plasma surface modification in bone tissue engineering[J]. Applied Surface Science, 2016 , 388(2016): 321-330.

[20] GAO F, XU Z Y, LIANG Q F, et al. Direct 3D printing of high strength biohybrid gradient hydrogel scaffolds for efficient repair of osteochondral defect[J]. Advanced Functional Materials, 2018, 28(13): 1-13.

[21] HA Y, YANG J, TAO F, et al. Phase-transited lysozyme as a universal route to bioactive hydroxyapatite crystalline film [J]. Advanced Functional Materials, 2018, 28(4): 1-12.

[22] ZHU C L, PONGKITWITOON S, QIU J C, et al. Design and fabrication of a hierarchically structured scaffold for tendon-to-bone repair[J]. Advanced Materials, 2018, 30(16): $1-8$.

[23] LIN Z F, WU M M, HE H M, et al. 3D printing of mechanically stable calcium-free alginate-based scaffolds with tunable surface charge to enable cell adhesion and facile biofunctionalization[J]. Advanced Functional Materials, 2019, 29(9): 1-14.

[24] SHAO J D, RUAN C S, XIE H H, et al. Black-phosphorus-incorporated hydrogel as a sprayable and biodegradable photothermal platform for postsurgical treatment of cancer[J]. Advanced Science, 2018，5(5): 1-11.

[25] ZHAI X Y, MA Y F, HOU C Y, et al. 3D-printed high strength bioactive supramolecular polymer/clay nanocomposite hydrogel scaffold for bone regeneration[J]. ACS Biomaterials Science \& Engineering, 2017, 3(6): 1109-1118.

[26] TARAFDER S, BOSE S. Polycaprolactone-coated 3D printed tricalcium phosphate scaffolds for bone tissue engineering: In vitro alendronate release behavior and local delivery effect on in vivo osteogenesis[J]. ACS Applied Materials \& Interfaces, 2014, 6(13): 9955-9965.

[27] SHARMA C, DINDA A K, POTDAR P D, et al. Fabrication and characterization of novel nano-biocomposite scaffold of chitosan-gelatin-alginate-hydroxyapatite for bone tissue engineering $[\mathrm{J}]$. Materials Science \& Engineering C-Materials for Biological Applications, 2016, 64(2016): 416-427.

[28] 李涤尘, 贺健康, 田小永, 等. 增材制造: 实现宏微结 构一体化制造 [J]. 机械工程学报, 2013，49(06)： 129-135.

LI Dichen, HE Jiankang, TIAN Xiaoyong, et al. Additive manufacturing : Integrated fabrication of macro/microstructures[J]. Journal of Mechanical Engineering , 2013, 49(06): 129-135.

[29] HE J K, LI D C, LIU Y X, et al. Preparation of chitosan-gelatin hybrid scaffolds with well-organized microstructures for hepatic tissue engineering[J]. Acta Biomaterialia, 2009, 5(1): 453-461.

[30] BIAN W G, LI D C, LIAN Q, et al. Fabrication of a bio-inspired beta-tricalcium phosphate/collagen scaffold based on ceramic stereolithography and gel casting for osteochondral tissue engineering $[\mathrm{J}]$. Rapid Prototyping Journal, 2012, 18(1): 68-80.

[31] 毛伟, 连芩, 李涤尘, 等. 立体空心血管网水凝胶支架 的 3D 打印工艺研究[J]. 机械工程学报, 2017, 53(09): 180-186.

MAO Wei, LIAN Qin, LI Dichen, et al. 3D printing process for hydro gel with the three-dimensional micro tubes to mimic vascular network[J]. Journal of Mechanical Engineering, 2017, 53(09): 180-186.

[32] 杨立军, 张佳, 王哲, 等. 组织工程骨支架内部微孔结 构流场特性分析 [J]. 机械工程学报，2018，54(20): 71-80.

YANG Lijun, ZHANG Jia, WANG Zhe, et al. Analysis of the flow field characteristics of micro-pore structure in tissue engineering scaffold $[\mathrm{J}]$. Journal of Mechanical 
Engineering, 2018, 54(20): 71-80.

[33] KIM H W, KNOWLES J C, KIM H E. Development of hydroxyapatite bone scaffold for controlled drug release via poly(epsilon-caprolactone) and hydroxyapatite hybrid coatings[J]. Journal of Biomedical Materials Research Part B-Applied Biomaterials，2004，70B(2): 240-249.

[34] KIM H W, KNOWLES J C, KIM H E. Hydroxyapatite porous scaffold engineered with biological polymer hybrid coating for antibiotic vancomycin release[J]. Journal of Materials Science-Materials in Medicine, 2005, 16(3): 189-195.

[35] LI Y, WU Z G, LI X K, et al. A polycaprolactone-tricalcium phosphate composite scaffold as an autograft-free spinal fusion cage in a sheep model[J]. Biomaterials, 2014, 35(22): 5647-5659.

[36] LIU X M, WU S L, YEUNG K W K, et al. Relationship between osseointegration and superelastic biomechanics in porous NiTi scaffolds[J]. Biomaterials，2011，32(2): 330-338.

[37] SPOERKE E D, MURRAY N G, LI H L, et al. A bioactive titanium foam scaffold for bone repair[J]. Acta Biomaterialia, 2005, 1(5): 523-533.

[38] WU S L, LIU X M, YEUNG K W K, et al. Biomimetic porous scaffolds for bone tissue engineering[J]. Materials Science \& Engineering R-Reports, 2014, 80(2014): 1-36.

[39] BELLER G, BURKHART M, FELSENBERG D, et al. Vertebral body data set ESA29-99-13 [EB/OL]. http: //bone3d.zib.de/data/2005/ESA29-99-L3/.

[40] GOMEZ S, VLAD M D, LOPEZ J, et al. Design and properties of 3D scaffolds for bone tissue engineering [J]. Acta Biomaterialia, 2016, 42(2016): 341-350.

[41] KANHED S, AWASTHI S, GOEL S, et al. Porosity distribution affecting mechanical and biological behaviour of hydroxyapatite bioceramic composites[J]. Ceramics International, 2017, 43(13): 10442-10449.

[42] 康建峰, 王玲, 孙畅宁, 等. 面向 3D 打印可变模量金 属假体的微结构设计[J]. 机械工程学报, 2017, 53(5): 175-180.

KANG Jianfeng, WANG Ling, SUN Changning, et al. Microstructure design for 3D printed metal prosthesis of adjustable modulus[J]. Journal of Mechanical Engineering, 2017, 53(5): 175-180.

[43] WANG G J, SHEN L D, ZHAO J F, et al. Design and compressive behavior of controllable irregular porous scaffolds: Based on voronoi-tessellation and for additive manufacturing $[\mathrm{J}]$. ACS Biomaterials Science \& Engineering, 2018, 4(2): 719-727.

[44] FRAZIER W E. Metal additive manufacturing : A review $[\mathrm{J}]$. Journal of Materials Engineering and
Performance, 2014, 23(6): 1917-1928.

[45] GAO W, ZHANG Y B, RAMANUJAN D, et al. The status, challenges, and future of additive manufacturing in engineering[J]. Computer-Aided Design, 2015, 69(2015): 65-89.

[46] GU D D, MEINERS W, WISSENBACH K, et al. Laser additive manufacturing of metallic components : Materials, processes and mechanisms [J]. International Materials Reviews, 2012, 57(3): 133-164.

[47] HUANG S H, LIU P, MOKASDAR A, et al. Additive manufacturing and its societal impact: A literature review $[\mathrm{J}]$. International Journal of Advanced Manufacturing Technology, 2013, 67(5-8): 1191-1203.

[48] MELCHELS F P W, DOMINGOS M A N, KLEIN T J, et al. Additive manufacturing of tissues and organs[J]. Progress in Polymer Science, 2012, 37(8): 1079-1104.

[49] VAEZI M, SEITZ H, YANG S F. A review on 3D micro-additive manufacturing technologies $[\mathrm{J}]$. International Journal of Advanced Manufacturing Technology, 2013, 67(5-8): 1721-1754.

[50] 王镓垠, 柴否, 刘利彪, 等. 人体器官 $3 \mathrm{D}$ 打印的最新 进展 [J]. 机械工程学报，2014，50(23)：119-127.

WANG Jiayin, CHAI Lei， LIU Libiao, et al. Progress in three-dimensional (3D) printing of artificial organs[J]. Journal of Mechanical Engineering, 2014, 50(23): 119-127.

[51] YAN Y N, WANG X H, XIONG Z, et al. Direct construction of a three-dimensional structure with cells and hydrogel[J]. Journal of Bioactive and Compatible Polymers, 2005, 20(3): 259-269.

[52] YAN Y N, WANG X H, PAN Y Q, et al. Fabrication of viable tissue-engineered constructs with 3D cell-assembly technique[J]. Biomaterials, 2005, 26(29): 5864-5871.

[53] XIONG Z, YAN Y N, ZHANG R J, et al. Fabrication of porous poly(l-lactic acid) scaffolds for bone tissue engineering via precise extrusion[J]. Scripta Materialia, 2001, 45(7): 773-779.

[54] CHUA C K, LEONG K F, CHEAH C M, et al. Development of a tissue engineering scaffold structure library for rapid prototyping. Part 1: Investigation and classification[J]. International Journal of Advanced Manufacturing Technology, 2003, 21(4): 291-301.

[55] CHUA C K, LEONG K F, CHEAH C M, et al. Development of a tissue engineering scaffold structure library for rapid prototyping. Part 2: Parametric library and assembly program[J]. International Journal of Advanced Manufacturing Technology, 2003, 21(4): 302-312.

[56] HOLLISTER S J. Porous scaffold design for tissue 
engineering $[\mathrm{J}]$. Nature Materials, 2005, 4(7): 518-524.

[57] KAPFER S C, HYDE S T, MECKE K, et al. Minimal surface scaffold designs for tissue engineering[J]. Biomaterials, 2011, 32(29): 6875-6882.

[58] YOO D J. Three-dimensional surface reconstruction of human bone using a b-spline based interpolation approach[J]. Computer-Aided Design, 2011，43(8): 934-947.

[59] YOO D J. Heterogeneous minimal surface porous scaffold design using the distance field and radial basis functions[J]. Medical Engineering \& Physics, 2012, 34(5): $625-639$.

[60] FENG J W, FU J Z, SHANG C, et al. Porous scaffold design by solid $\mathrm{T}$-splines and triply periodic minimal surfaces[J]. Computer Methods in Applied Mechanics and Engineering, 2018, 336(2018): 333-352.

[61] LUO Y X, LI Y X, QIN X L, et al. 3D printing of concentrated alginate/gelatin scaffolds with homogeneous nano apatite coating for bone tissue engineering $[\mathrm{J}]$. Materials \& Design, 2018, 146(2018): 12-19.
[62] JANG C H, LEE J, KIM G. Synergistic effect of alginate/BMP-2/umbilical cord serum-coated on 3D-printed PCL biocomposite for mastoid obliteration model $[\mathrm{J}]$. Journal of Industrial and Engineering Chemistry, 2019, 72(2019): 432-441.

[63] PAN S, ZHONG Y, SHAN Y B, et al. Selection of the optimum 3D-printed pore and the surface modification techniques for tissue engineering tracheal scaffold in vivo reconstruction[J]. Journal of Biomedical Materials Research Part A， 2019， 107(2): 360-370.

作者简介: 屈华伟, 男, 1991 年出生, 博士研究生。主要研究方向为骨 组织工程生物支架设计和 3D 打印。

E-mail: quhuawei@hit.edu.cn

韩振宇, 男, 1978 年出生, 博士, 教授, 博士研究生导师。主要研究方 向为骨骼支架 $3 \mathrm{D}$ 打印成型、复合材料缠绕成型 $\mathrm{CAD} / \mathrm{CAM}$ 技术、智能 加工技术和自动铺丝技术等。

E-mail: hanzy@hit.edu.cn

富宏亚(通信作者), 男, 1963 年出生, 博士, 教授, 博士研究生导师。 主要研究方向为生物 3D 打印、纤维铺放技术、智能加工技术和非标数 控设备研制等。

E-mail: hongyafu@hit.edu.cn 